\title{
Synthesis and Analysis of Product-form Petri Nets
}

\author{
S. Haddad ${ }^{1}$, J. Mairesse ${ }^{2}$, H-T. Nguyen ${ }^{2}$ \\ 1 ENS Cachan, LSV, CNRS UMR 8643, INRIA, Cachan, France \\ haddad@lsv.ens-cachan.fr \\ 2 Université Paris 7, LIAFA, CNRS UMR 7089, Paris, France \\ \{mairesse, ngthach\}@liafa.jussieu.fr
}

\begin{abstract}
For a large Markovian model, a "product form" is an explicit description of the steady-state behaviour which is otherwise generally untractable. Being first introduced in queueing networks, it has been adapted to Markovian Petri nets. Here we address three relevant issues for product-form Petri nets which were left fully or partially open: (1) we provide a sound and complete set of rules for the synthesis; (2) we characterise the exact complexity of classical problems like reachability; (3) we introduce a new subclass for which the normalising constant (a crucial value for product-form expression) can be efficiently computed.
\end{abstract}

Keywords: Petri nets, product-form, synthesis, complexity analysis, reachability, normalising constant

\section{Introduction}

Product-form for stochastic models. Markovian models of discrete events systems are powerful formalisms for modelling and evaluating the performances of such systems. The main goal is the equilibrium performance analysis. It requires to compute the stationary distribution of a continuous time Markov process derived from the model. Unfortunately the potentially huge (sometimes infinite) state space of the models often prevents the modeller from computing explicitly this distribution. To cope with the issue, one can forget about exact solutions and settle for approximations, bounds, or even simulations. The other possibility is to focus on subclasses for which some kind of explicit description is indeed possible. In this direction, the most efficient and satisfactory approach may be the product-form method: for a model composed of modules, the stationary probability of a global state may be expressed as a product of quantities depending only on local states divided by a normalising constant.

Such a method is applicable when the interactions between the modules are "weak". This is the case for queueing networks where the interactions between queues are described by a random routing of clients. Various classes of queueing networks with product-form solutions have been exhibited [11, 3, 12]. Moreover efficient algorithms have been designed for the computation of the normalising constant [18]. 
Product-form Petri nets. Due to the explicit modelling of competition and synchronisation, the Markovian Petri nets formalism [1] is an attractive modelling paradigm. Similarly to queueing networks, product-form Markovian Petri Nets were introduced to cope with the combinatorial explosion of the state space. Historically, works started with purely behavioural properties (i.e. by an analysis of the reachability graph) as in [13], and then progressively moved to more and more structural characterisations [14, 10]. Building on the work of [10], the authors of [9] establish the first purely structural condition for which a product form exists and propose a polynomial time algorithm to check for the condition, see also [15] for an alternative characterisation. These nets are called $\Pi^{2}$-nets.

\section{Open issues related to product-form Petri nets.}

- From a modelling point of view, it is more interesting to design specific types of Petri nets by modular constructions rather than checking a posteriori whether a net satisfies the specification. For instance, in [7], a sound and complete set of rules is proposed for the synthesis of live and bounded freechoice nets. Is it possible to get an analog for product-form Petri nets?

- From a qualitative analysis point of view, it is interesting to know the complexity of classical problems (reachability, coverability, liveness, etc.) for a given subclass of Petri nets and to compare it with that of general Petri nets. For product-form Petri nets, partial results were presented in [9] but several questions were left open. For instance, the reachability problem is PSPACE-complete for safe Petri nets but in safe product-form Petri nets it is only proved to be NP-hard in [9].

- From a quantitative analysis point of view, an important and difficult issue is the computation of the normalising constant. Indeed, in product-form Petri nets, one can directly compute relative probabilities (e.g. available versus unavailable service), but determining absolute probabilities requires to compute the normalising constant (i.e. the sum over reachable states of the relative probabilities). In models of queueing networks, this can be efficiently performed using dynamic programming. In Petri nets, it has been proved that the efficient computation is possible when the linear invariants characterise the set of reachable markings [6]. Unfortunately, all the known subclasses of product-form nets that fulfill this characterisation are models of queueing networks!

Our contribution. Here we address the three above issues. In Section 3, we provide a set of sound and complete rules for generating any $\Pi^{2}$-net. We also use these rules for transforming a general Petri net into a related product-form Petri net. In Section 4, we solve relevant complexity issues. More precisely, we show that the reachability and liveness problems are PSPACE-complete for safe product-form nets and that the coverability problem is EXPSPACE-complete for general product-form nets. From these complexity results, we conjecture that the problem of computing the normalising constant does not admit an efficient solution for the general class of product-form Petri nets. However, in Section 5 , we introduce a large subclass of product-form Petri nets, denoted $\Pi^{3}$-nets, for which the normalising constant can be efficiently computed. We emphasise 
that contrary to all subclasses related to queueing networks, $\Pi^{3}$-nets may admit spurious markings (i.e. that fufill the invariants while being unreachable).

The above results may change our perspective on product-form Petri nets. It is proved in [15] that the intersection of free-choice and product-form Petri nets is the class of Jackson networks [11]. This may suggest that the class of productform Petri nets is somehow included in the class of product-form queueing networks. In the present paper, we refute this belief in two ways. First by showing that some classical problems are as complex for product-form Petri nets as for general Petri nets whereas they become very simple for product-form queueing networks. Second by exhibiting the class of $\Pi^{3}$-nets, see the above discussion.

A version of the present paper including proofs can be found on arXiv.

Notations. We often denote a vector $u \in \mathbb{R}^{S}$ by $\sum_{s} u(s) s$. The support of vector $u$ is the subset $S^{\prime} \equiv\{s \in S \mid u(s) \neq 0\}$.

\section{Petri nets, product-form nets, and $\Pi^{2}$-nets}

Definition 2.1 (Petri net) $A$ Petri net is a 5-tuple $\mathcal{N}=\left(P, T, W^{-}, W^{+}, m_{0}\right)$ where:

- $P$ is a finite set of places;

- $T$ is a finite set of transitions, disjoint from $P$;

$-W^{-}$, resp. $W^{+}$, is a $P \times T$ matrix with coefficients in $\mathbb{N}$;

$-m_{0} \in \mathbb{N}^{P}$ is the initial marking.

Below, we also call Petri net the unmarked quadruple $\left(P, T, W^{-}, W^{+}\right)$. The presence or absence of a marking will depend on the context.

A Petri net is represented in Figure 1. The following graphical conventions are used: places are represented by circles and transitions by rectangles. There is an arc from $p \in P$ to $t \in T$ (resp. from $t \in T$ to $p \in P$ ) if $W^{+}(p, t)>0$ (resp. $\left.W^{-}(p, t)>0\right)$, and the weight $W^{+}(p, t)$ (resp. $\left.W^{-}(p, t)\right)$ is written above the corresponding arc except when it is equal to 1 in which case it is omitted. The initial marking is materialised: if $m_{0}(p)=k$, then $k$ tokens are drawn inside the circle $p$. Let $P^{\prime} \subset P$ and $m$ be a marking then $m\left(P^{\prime}\right)$ is defined by $m\left(P^{\prime}\right) \equiv \sum_{p \in P^{\prime}} m(p)$.

The matrix $W=W^{+}-W^{-}$is the incidence matrix of the Petri net. The input bag ${ }^{\bullet} t$ (resp. output bag $t^{\bullet}$ ) of the transition $t$ is the column vector of $W^{-}$(resp. $W^{+}$) indexed by $t$. For a place $p$, we define $\bullet^{\bullet} p$ and $p^{\bullet}$ similarly. A $T$-semi-flow (resp. $S$-semi-flow) is a $\mathbb{Q}$-valued vector $v$ such that $W . v=0$ (resp. $v . W=0)$.

A symmetric Petri net is a Petri net such that: $\forall t \in T, \exists t^{-} \in T, \quad{ }^{\bullet} t=$ $\left(t^{-}\right)^{\bullet}, t^{\bullet}={ }^{\bullet} t^{-}$. A state machine is a Petri net such that: $\forall t \in T,|\bullet t|=\left|t^{\bullet}\right|=1$.

Definition 2.2 (Firing rule) A transition $t$ is enabled by the marking $m$ if $m \geq{ }^{\bullet} t$ (denoted by $m \stackrel{t}{\longrightarrow}$ ); an enabled transition $t$ may fire which transforms the marking $m$ into $m-\bullet t+t^{\bullet}$, denoted by $m \stackrel{t}{\longrightarrow} m^{\prime}=m-\bullet t+t^{\bullet}$. 


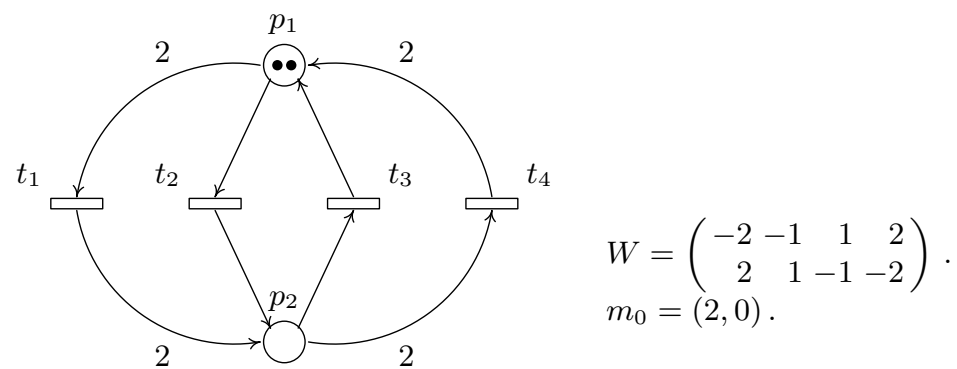

Fig. 1. Petri net.

A marking $m^{\prime}$ is reachable from the marking $m$ if there exists a firing sequence $\sigma=t_{1} \ldots t_{k}(k \geq 0)$ and a sequence of markings $m_{1}, \ldots, m_{k-1}$ such that $m \stackrel{t_{1}}{\rightarrow}$ $m_{1} \stackrel{t_{2}}{\longrightarrow} \cdots \stackrel{t_{k-1}}{\longrightarrow} m_{k-1} \stackrel{t_{k}}{\rightarrow} m^{\prime}$. We write in a condensed way: $m \stackrel{\sigma}{\rightarrow} m^{\prime}$.

We denote by $\mathcal{R}(m)$ the set of markings which are reachable from the marking $m$. The reachability graph of a Petri net with initial marking $m_{0}$ is the directed graph with nodes $\mathcal{R}\left(m_{0}\right)$ and $\operatorname{arcs}\left\{\left(m, m^{\prime}\right) \mid \exists t \in T: m \stackrel{t}{\rightarrow} m^{\prime}\right\}$.

Given $\left(\mathcal{N}, m_{0}\right)$ and $m_{1}$, the reachability problem is to decide if $m_{1} \in \mathcal{R}\left(m_{0}\right)$, and the coverability problem is to decide if $\exists m_{2} \in \mathcal{R}\left(m_{0}\right), m_{2} \geq m_{1}$.

A Petri net $\left(\mathcal{N}, m_{0}\right)$ is live if every transition can always be enabled again, that is: $\forall m \in \mathcal{R}\left(m_{0}\right), \forall t \in T, \exists m^{\prime} \in \mathcal{R}(m), m^{\prime} \stackrel{t}{\rightarrow}$. A Petri net $\left(\mathcal{N}, m_{0}\right)$ is bounded if $\mathcal{R}\left(m_{0}\right)$ is finite. It is safe or 1-bounded if: $\forall m \in \mathcal{R}\left(m_{0}\right), m(p) \leq 1$.

\subsection{Product-form Petri nets}

There exist several ways to define timed models of Petri nets, see [2]. We consider the model of Markovian Petri nets with race policy. Roughly, with each enabled transition is associated a "countdown clock" whose positive initial value is set at random according to an exponential distribution whose rate depends on the transition. The first transition to reach 0 fires, which may enable new transitions and start new clocks.

Definition 2.3 (Markovian PN) A Markovian Petri net (with race policy) is a Petri net equipped with a set of rates $\left(\mu_{t}\right)_{t \in T}, \mu_{t} \in \mathbb{R}_{+} \backslash\{0\}$. The firing time of an enabled transition $t$ is exponentially distributed with parameter $\mu_{t}$. The marking evolves as a continuous-time jump Markov process with state space $\mathcal{R}\left(m_{0}\right)$ and infinitesimal generator $Q=\left(q_{m, m^{\prime}}\right)_{m, m^{\prime} \in \mathcal{R}\left(m_{0}\right)}$, given by:

$$
\forall m, \forall m^{\prime} \neq m, q_{m, m^{\prime}}=\sum_{t: m \stackrel{t}{\rightarrow} m^{\prime}} \mu_{t}, \quad \forall m, q_{m, m}=-\sum_{m^{\prime} \neq m} q_{m, m^{\prime}} .
$$

W.l.o.g., we assume that there is no transition $t$ such that ${ }^{\bullet} t=t^{\bullet}$. Indeed, the firing of such a transition does not modifiy the marking, so its removal does not modify the infinitesimal generator. We also assume that $\left(\bullet^{\bullet} t_{1} t_{1}^{\bullet}\right) \neq\left(\bullet_{2}, t_{2}^{\bullet}\right)$ 
for all transitions $t_{1} \neq t_{2}$. Indeed, if it is not the case, the two transitions may be replaced by a single one with the summed rate.

An invariant measure is a non-trivial solution $\nu$ to the balance equations: $\nu Q=0$. A stationary measure (distribution) $\pi$ is an invariant probability measure: $\pi Q=0, \sum_{m} \pi(m)=1$.

Definition 2.4 (Product-form PN) A Petri net is a product-form Petri net if for all rates $\left(\mu_{t}\right)_{t \in T}$, the corresponding Markovian Petri net admits an invariant measure $\nu$ satisfying:

$$
\exists\left(u_{p}\right)_{p \in P}, u_{p} \in \mathbb{R}_{+}, \quad \forall m \in \mathcal{R}\left(m_{0}\right), \quad \nu(m)=\prod_{p \in P} u_{p}^{m_{p}} .
$$

The existence of $\nu$ satisfying (2.2) implies that the marking process is irreducible (in other words, the reachability graph is strongly connected). In (2.2), the mass of the measure, i.e. $\nu\left(\mathcal{R}\left(m_{0}\right)\right)=\sum_{m} \nu(m)$, may be either finite or infinite. For a bounded Petri net, the mass is always finite. But for an unbounded Petri net, the typical situation will be as follows: structural conditions on the Petri net will ensure that the Petri net is a product-form one. Then, for some values of the rates, $\nu$ will have an infinite mass, and, for others, $\nu$ will have a finite mass. In the first situation, the marking process will be either transient or recurrent null (unstable case). In the second situation, the marking process will be positive recurrent (stable or ergodic case).

When the mass is finite, we call $\nu\left(\mathcal{R}\left(m_{0}\right)\right)$ the normalising constant. The probability measure $\pi(\cdot)=\nu\left(\mathcal{R}\left(m_{0}\right)\right)^{-1} \nu(\cdot)$ is the unique stationary measure of the marking process. Computing explicitly the normalising constant is an important issue, see Section 5.

The goal is now to get sufficient conditions for a Petri net to be of productform. To that purpose, we introduce three notions: weak reversibility, deficiency, and witnesses.

Let $\left(N, m_{0}\right)$ be a Petri net. The set of complexes is defined by $\mathcal{C}=\{\bullet t \mid t \in$ $T\} \cup\left\{t^{\bullet} \mid t \in T\right\}$. The reaction graph is the directed graph whose set of nodes is $\mathcal{C}$ and whose set of $\operatorname{arcs}$ is $\{(\bullet, t \bullet) \mid t \in T\}$.

Definition 2.5 (Weak reversibility: П-nets) A Petri net is weakly reversible (WR) if every connected component of its reaction graph is strongly connected. Weakly reversible Petri nets are also called $\Pi$-nets.

The notion and the name "WR" come from the chemical literature. In the Petri net context, it was introduced in [4, Assumption 3.2] under a different name and with a slightly different but equivalent formulation. WR is a strong constraint. It should not be confused with the classical notion of "reversibility" (the marking graph is strongly connected). In particular, WR implies reversibility! Observe that all symmetric Petri nets are WR.

The notion of deficiency is due to Feinberg [8]. 
Definition 2.6 (Deficiency) Consider a Petri net with incidence matrix $W$ and set of complexes $\mathcal{C}$. Let $\ell$ be the number of connected components of the reaction graph. The deficiency of the Petri net is defined by: $|\mathcal{C}|-\ell-\operatorname{rank}(W)$.

The notion of witnesses appears in [9].

Definition 2.7 (Witness) Let c be a complex. A witness of $c$ is a vector wit $(c) \in$ $\mathbb{Q}^{P}$ such that for all transition $t$ :

$$
\begin{cases}\text { wit }(c) \cdot W(t)=-1 & \text { if } \bullet=c \\ \text { wit }(c) \cdot W(t)=1 & \text { if } t^{\bullet}=c \\ \text { wit }(c) \cdot W(t)=0 & \text { otherwise }\end{cases}
$$

where $W(t)$ denotes the column vector of $W$ indexed by $t$.

Examples. Consider the Petri net of Figure 1. First, it is WR. Indeed, the set of complexes is $\mathcal{C}=\left\{p_{1}, p_{2}, 2 p_{1}, 2 p_{2}\right\}$ and the reaction graph is:

$$
p_{1} \leftrightarrow p_{2}, 2 p_{1} \leftrightarrow 2 p_{2},
$$

with two connected components which are strongly connected. Second, the deficiency is 1 since $|\mathcal{C}|=4, \ell=2$, and $\operatorname{rank}(W)=1$. Last, one can check that none of the complexes admit a witness.

Consider now the Petri net of Figure 3. It is WR and it has deficiency 0.

Proposition 2.8 (deficiency $0 \Longleftrightarrow$ witnesses, in [15, Prop. 3.9]) A Petri net admits a witness for each complex iff it has deficiency 0.

Next Theorem is a combination of Feinberg's Deficiency zero Theorem [8] and Kelly's Theorem [12, Theorem 8.1]. (It is proved under this form in [15, Theorem 3.8].)

Theorem 2.9 (WR + deficiency $0 \Longrightarrow$ product-form) Consider a Markovian Petri net with rates $\left(\mu_{t}\right)_{t \in T}, \mu_{t}>0$, and assume that the underlying Petri net is $W R$ and has deficiency 0 . Then there exists $\left(u_{p}\right)_{p \in P}, u_{p}>0$, satisfying the equations:

$$
\forall c \in \mathcal{C}, \quad \prod_{p: c_{p} \neq 0} u_{p}^{c_{p}} \sum_{t: \bullet t=c} \mu_{t}=\sum_{t: t^{\bullet}=c} \mu_{t} \prod_{p: \bullet t_{p} \neq 0} u_{p}^{\bullet_{p}}
$$

The marking process has an invariant measure $\nu$ s.t.: $\forall m, \nu(m)=\prod_{p \in P} u_{p}^{m_{p}}$.

Checking the WR, computing the deficiency, determining the witnesses, and solving the equations (2.3), all of these operations can be performed in polynomialtime, see $[9,15]$.

Summing up the above, it seems worth to isolate and christen the class of nets which are WR and have deficiency 0 . We adopt the terminology of [9].

Definition $2.10\left(\Pi^{2}\right.$-net) $A \Pi^{2}$-net is a Petri net which is WR and has deficiency 0 . 


\section{Synthesis and regulation of $\Pi^{2}$-nets}

The reaction graph, defined in Section 2.1, may be viewed as a Petri net (state machine). Let us formalise this observation. The reaction Petri net of $\mathcal{N}$ is the Petri net $\mathcal{A}=\left(\mathcal{C}, T, \bar{W}^{-}, \bar{W}^{+}\right)$, with for every $t \in T$ :

$-\bar{W}^{-}(\bullet t, t)=1$ and $\forall u \neq \bullet t, \bar{W}^{-}(u, t)=0$

$-\bar{W}^{+}\left(t^{\bullet}, t\right)=1$ and $\forall u \neq t^{\bullet}, \bar{W}^{+}(u, t)=0$

\subsection{Synthesis}

In this subsection, we consider unmarked nets. We define three rules that generate all the $\Pi^{2}$-nets. The first rule adds a strongly connected state machine.

Definition 3.1 (State-machine insertion) Let $\mathcal{N}=\left(P_{\mathcal{N}}, T_{\mathcal{N}}, W_{\mathcal{N}}^{-}, W_{\mathcal{N}}^{+}\right)$be a net and $\mathcal{M}=\left(P_{\mathcal{M}}, T_{\mathcal{M}}, W_{\mathcal{M}}^{-}, W_{\mathcal{M}}^{+}\right)$be a strongly connected state machine disjoint from $\mathcal{N}$. The rule $\mathrm{S}-$ add is always applicable and $\mathcal{N}^{\prime}=\mathrm{S}-\operatorname{add}(\mathcal{N}, \mathcal{M})$ is defined by:

- $P^{\prime}=P_{\mathcal{N}} \sqcup P_{\mathcal{M}}, T^{\prime}=T_{\mathcal{N}} \sqcup T_{\mathcal{M}} ;$

$-\forall p \in P_{\mathcal{N}}, \forall t \in T_{\mathcal{N}}, W^{\prime-}(p, t)=W_{\mathcal{N}}^{-}(p, t), W^{\prime+}(p, t)=W_{\mathcal{N}}^{+}(p, t) ;$

$-\forall p \in P_{\mathcal{M}}, \forall t \in T_{\mathcal{M}}, W^{\prime-}(p, t)=W_{\mathcal{M}}^{-}(p, t), W^{\prime+}(p, t)=W_{\mathcal{M}}^{+}(p, t) ;$

- All other entries of $W^{\prime-}$ and $W^{\prime+}$ are null.

The second rule consists in substituting to a complex $c$ the complex $c+\lambda p$. However in order to be applicable some conditions must be fulfilled. The first condition requires that $c(p)+\lambda$ is non-negative. The second condition ensures that the substitution does not modify the reaction graph. The third condition preserves deficiency zero. Observe that the third condition can be checked in polynomial time, indeed it amounts to solving a system of linear equations in $\mathbb{Q}$ for every complex.

Definition 3.2 (Complex update) Let $\mathcal{N}=\left(P, T, W^{-}, W^{+}\right)$be a $\Pi^{2}$-net, $c$ be a complex of $\mathcal{N}, p \in P, \lambda \in \mathbb{Z}^{*}$. The rule C-update is applicable when:

1. $\lambda+c(p) \geq 0$;

2. $c+\lambda p$ is not a complex of $\mathcal{N}$;

3. For every complex $c^{\prime}$ there exists a witness wit $\left(c^{\prime}\right)$ s.t. wit $\left(c^{\prime}\right)(p)=0$.

The resulting net $\mathcal{N}^{\prime}=\mathrm{C}$-update $(\mathcal{N}, c, p, \lambda)$ is defined by:

$-P^{\prime}=P, T^{\prime}=T$;

$-\forall t \in T$ s.t. $W^{-}(t) \neq c, W^{-}(t)=W^{-}(t), \forall t \in T$ s.t. $W^{-}(t)=c, W^{\prime-}(t)=c+\lambda p$

$-\forall t \in T$ s.t. $W^{+}(t) \neq c, W^{\prime+}(t)=W^{-}(t), \forall t \in T$ s.t. $W^{+}(t)=c, W^{+}(t)=c+\lambda p$.

The last rule "cleans" the net by deleting an isolated place. We call this operation P-delete.

Definition 3.3 (Place deletion) Let $\mathcal{N}=\left(P, T, W^{-}, W^{+}\right)$be a net and let $p$ be an isolated place of $\mathcal{N}$, i.e. $W^{-}(p)=W^{+}(p)=0$. Then the rule $\mathrm{P}$-delete is applicable and $\mathcal{N}^{\prime}=\mathrm{P}-\operatorname{delete}(\mathcal{N}, p)$ is defined by: 
$-P^{\prime}=P \backslash\{p\}, T^{\prime}=T$;

$-\forall q \in P^{\prime}, W^{\prime-}(q)=W^{-}(q), W^{\prime+}(q)=W^{+}(q)$.

Proposition 3.4 shows the interest of the rules for synthesis of $\Pi^{2}$-nets.

Proposition 3.4 (Soundness and Completeness) Let $\mathcal{N}$ be $a \Pi^{2}$-net.

- If a rule $\mathrm{S}$-add, C-update or $\mathrm{P}$-delete is applicable on $\mathcal{N}$ then the resulting net is still $a \Pi^{2}$-net.

- The net $\mathcal{N}$ can be obtained by successive applications of the rules $\mathrm{S}$-add, $\mathrm{C}$-update, $\mathrm{P}$-delete starting from the empty net.

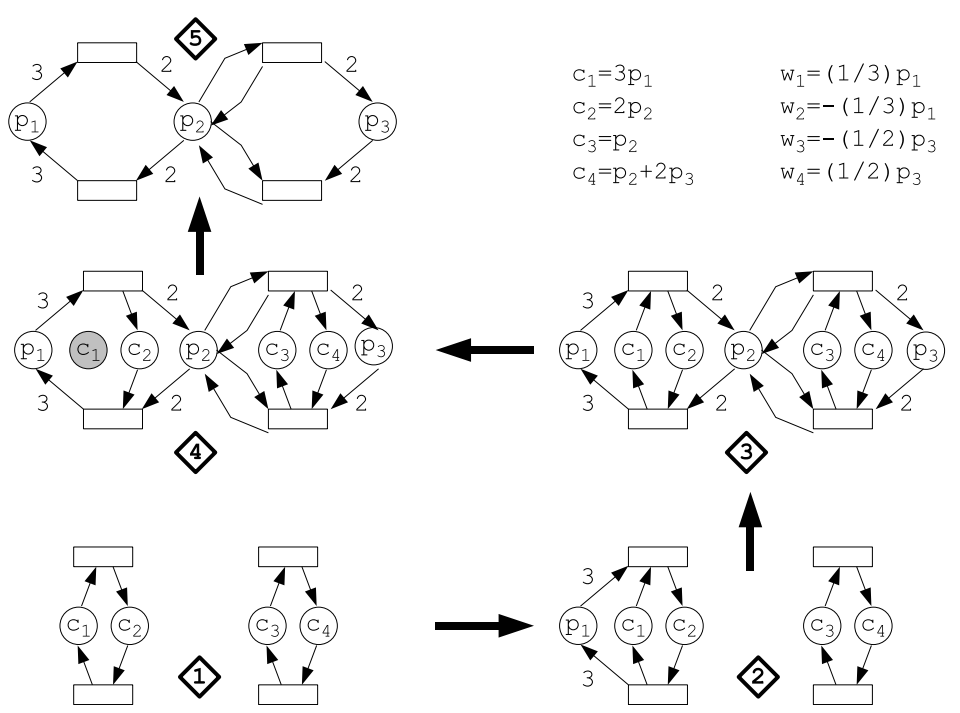

Fig. 2. How to synthetise a $\Pi^{2}$-net.

We illustrate the synthesis process using our rules on the net numbered 5 in Figure 2. We have also indicated on the right upper part of this figure, the four complexes and their witnesses. Since the reaction Petri graph of this net has two state machines, we start by creating it using twice the insertion of a state machine (net 1 ). Then we add the place $p_{1}$ (a particular state machine). We update the complex $c_{1}$ (the single one where $p_{1}$ appears in the original net) by adding $3 p_{1}$ (net 2). The new complex cannot appear elsewhere due to the presence of $c_{1}$. Iterating this process, we obtain the net 3 . Observe that this net is a fusion (via $T$ the set of transitions) of the original net and its reaction Petri net. We now iteratively update the complexes. The net 4 is the result of transforming $c_{1}+3 p_{1}$ into $3 p_{1}$. This transformation is applicable since all the 
complexes are witnessed by witnesses of the original net. For instance, $c_{1}+3 p_{1}$ is witnessed by $(1 / 3) p_{1}$. Once $c_{1}$ is isolated, we delete it. Iterating this process yields the original net.

For modelling purposes, we could define more general rules like the refinement of a place by a strongly connected state machine. Here the goal was to design a minimal set of rules.

\subsection{From non $\Pi^{2}$-nets to $\Pi^{2}$-nets}

Below we propose a procedure which takes as input any Petri net and returns a $\Pi^{2}$-net. The important disclaimer is that the resulting net, although related to the original one, has a different structural and timed behaviour. So it is up to the modeller to decide if the resulting net satisfies the desired specifications. In case of a positive answer, the clear gain is that all the associated Markovian Petri nets have a product form.

Consider a Petri net $\mathcal{N}=\left(P, T, W^{-}, W^{+}, m_{0}\right)$ with set of complexes $\mathcal{C}$. Assume that $\mathcal{N}$ is not WR. For each transition $t$, add a reverse transition $t^{-}$ such that ${ }^{\bullet} t^{-}=t^{\bullet}$ and $\left(t^{-}\right)^{\bullet}={ }^{\bullet} t$ (unless such a transition already exists). The resulting net is WR. In the Markovian Petri net, the added reverse transitions can be given very small rates, to approximate more closely the original net.

Now, to enforce deficiency 0, the idea is to compose a general Petri net with its reaction graph as in the illustration of Proposition 3.4.

Definition 3.5 Consider a Petri net $\mathcal{N}=\left(P, T, W^{-}, W^{+}, m_{0}\right)$. Let $\bar{m}_{0}$ be an initial marking for the reaction Petri net $\mathcal{A}$. The regulated Petri net associated with $\mathcal{N}$ is defined as follows:

$\mathcal{A} \odot \mathcal{N}=\left(P \sqcup \mathcal{C}, T, \widetilde{W}^{-}, \widetilde{W}^{+},\left(m_{0}, \bar{m}_{0}\right)\right), \quad \widetilde{W}^{-}=\left[\begin{array}{l}W^{-} \\ \bar{W}^{-}\end{array}\right], \quad \widetilde{W}^{+}=\left[\begin{array}{l}W^{+} \\ \bar{W}^{+}\end{array}\right]$.

Proposition 3.6 The regulated Petri net $\mathcal{A} \odot \mathcal{N}$ is WR iff $\mathcal{N}$ is $W R$. The regulated Petri net $\mathcal{A} \odot \mathcal{N}$ has deficiency 0 .

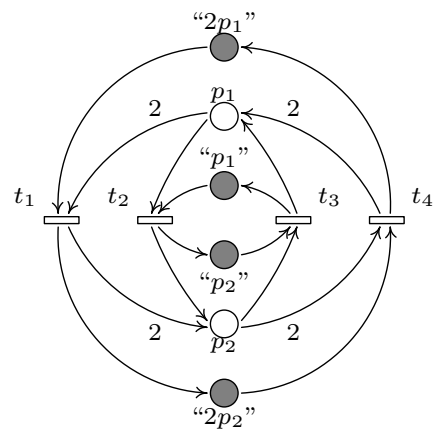

Fig. 3. Regulated Petri net associated with the Petri net of Fig 1. 
The behaviours of the original and regulated Petri nets are different. In particular, the regulated Petri net is bounded, even if the original Petri net is unbounded. Roughly, the regulation imposes some control on the firing sequences. Consider the example of Figures 1 (original net) and 3 (regulated net). The transitions $t_{1}$ and $t_{4}$ belong to the same simple circuit in the reaction graph. Let $w$ be an arbitrary firing sequence. The quantity $|w|_{t 1}-|w|_{t 4}$ is unbounded for the original net, and bounded for the regulated net.

\section{Complexity analysis of $\Pi^{2}$-nets}

All the nets that we build in this section are symmetric hence WR. For every depicted transition $t$, the reverse transition exists (sometimes implicitly) and is denoted $t^{-}$. It is well known that reachability and liveness of safe Petri nets are PSPACE-complete [5]. In [9], it is proved that reachability and liveness are PSPACE-hard for safe $\Pi$-nets and NP-hard for safe $\Pi^{2}$-nets. Next theorem and its corollary improve on these results by showing that the problem is not easier for safe $\Pi^{2}$-nets than for general safe Petri nets.

Theorem 4.1 The reachability problem for safe $\Pi^{2}$-nets is PSPACE-complete.

Proof. Our proof of PSPACE-hardness is based on a reduction from the QSAT problem [17]. QSAT consists in deciding whether the following formula is true

$$
\varphi \equiv \forall x_{n} \exists y_{n} \forall x_{n-1} \exists y_{n-1} \ldots \forall x_{1} \exists y_{1} \psi
$$

where $\psi$ is a propositional formula over $\left\{x_{1}, y_{1} \ldots, x_{n}, y_{n}\right\}$ in conjunctive normal form with at most three literals per clause.

Observe that in order to check the truth of $\varphi$, one must check the truth of $\psi$ w.r.t. the $2^{n}$ interpretations of $x_{1}, \ldots, x_{n}$ while the corresponding interpretation of any $y_{i}$ must only depend of the interpretation of $\left\{x_{n}, \ldots, x_{i}\right\}$.

Counters modelling. First we design a $\Pi^{2}$-net $\mathcal{N}_{\text {cnt }}$ that "counts" from 0 to $2^{k}-1$. This net is defined by:

- $P=\left\{p_{0}, \ldots, p_{k-1}, q_{0}, \ldots, q_{k-1}\right\}$;

$-T=\left\{t_{0}, \ldots, t_{k-1}\right\}$

- For every $0 \leq i<k, \bullet^{\bullet} t_{i}=p_{i}+\sum_{j<i} q_{j}$ and $t_{i}^{\bullet}=q_{i}+\sum_{j<i} p_{j}$;

- For every $0 \leq i<k, m_{0}\left(p_{i}\right)=1$ and $m_{0}\left(q_{i}\right)=0$.



Fig. 4. A 3-bit counter (without the reverse transitions). 
Observe that for every reachable marking $m$ and every index $i$, we have $m\left(p_{i}\right)+$ $m\left(q_{i}\right)=1$. Therefore $m$ can be coded by the binary word $\omega=\omega_{k-1} \ldots \omega_{0}$ in which $\omega_{i}=m\left(q_{i}\right)$. The word $\omega$ is interpreted as the binary expansion of an integer between 0 and $2^{k}-1$. We denote by $\operatorname{val}(\omega)$ the integer value associated with $w$. Consider $w \notin\left\{0^{k}, 1^{k}\right\}$, there are two markings reachable from $w$ which are $w+$ and $w-\operatorname{such}$ that $\operatorname{val}(w-)=\operatorname{val}(w)-1$ and $\operatorname{val}(w+)=\operatorname{val}(w)+1$.

The figure below represents the reachability graph of the 3-bit counter. For a $k$-bit counter, the shortest firing sequence from $0^{k}$ to $1^{k}$ is $\sigma_{k}$ defined inductively by: $\sigma_{1}=t_{0}$ and $\sigma_{i+1}=\sigma_{i} t_{i} \sigma_{i}$.



For every complex $c \equiv p_{i}+\sum_{j<i} q_{j}$ (resp. $c \equiv q_{i}+\sum_{j<i} p_{j}$ ), a possible witness is $w i t(c) \equiv p_{i}+\sum_{j>i} 2^{j-i-1} p_{j}$ (resp. wit $(c) \equiv q_{i}+\sum_{j>i} 2^{j-i-1} q_{j}$ ). Thus this subnet has deficiency 0 .

To manage transition firings between the update of counters, we duplicate the counter subnet and we synchronize the two subnets as indicated in the figure below. For a duplicated $k$-bit counter, the shortest firing sequence from the marking with the two counters set to $0^{k}$ and place go marked to the marking with the two counters set to $1^{k}$ and place $g o$ marked is obtained by: $\bar{\sigma}_{1}=\bar{t}_{0}$ and $\bar{\sigma}_{n+1}=\bar{\sigma}_{n} \bar{t}_{n} \bar{\sigma}_{n}$ where $\bar{t}_{i}=t_{i} t_{i}^{\prime}$.

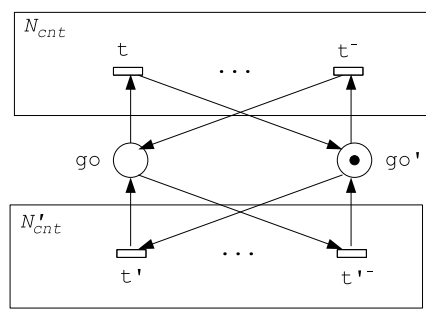

This net has still deficiency 0 since the complexes are just enlarged by the places go or $g o^{\prime}$ and their witnesses remain the same.

Variable modelling. For reasons that will become clear later on, the two counter subnets contain $n+3$ bits indexed from 0 to $n+2$. The bits $1, \ldots, n$ of counter $c$ t correspond to the value of variables $x_{1}, \ldots, x_{n}$. Managing the value of variables $y_{1}, \ldots, y_{n}$ is done as follows. For every variable $y_{i}$, we add the subnet described below on the left (observe that $s_{i}=r_{i}^{-1}$ ) and modify the two counter subnets as described on the right. 

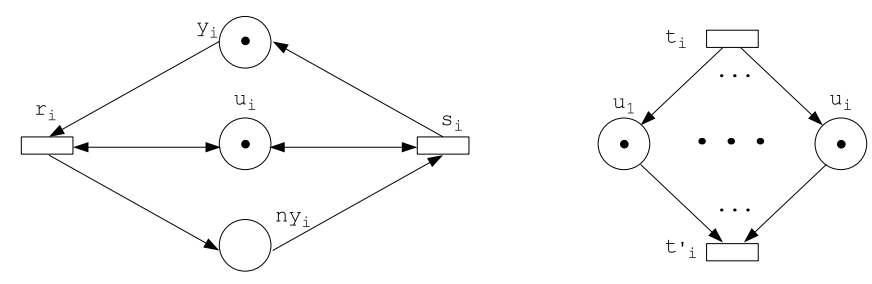

When place $y_{i}$ (resp. $n y_{i}$ ) is marked, this corresponds to interpreting variable $y_{i}$ as true (resp. false). Changes of the interpretation are possible when place $u_{i}$ is marked. This is the role of the modification done on the counter subnet: between a firing of $t_{i}$ and $t_{i}^{\prime}$ places $\left\{u_{j}\right\}_{j \leq i}$ are marked. With this construction, we get the expected behaviour: the interpretation of a variable $y_{i}$ can only be modified when the interpretation of a variable $x_{j}$ with $j \geq i$ is modified. The complexes of the counter subnet are enlarged with places $u_{i}$ and their witnesses remain the same since places in the support of these witnesses are not modified by transitions $s_{i}$ and $r_{i}$. The new complex $y_{i}+u_{i}$ (resp. $n y_{i}+u_{i}$ ) has for witness $y_{i}$ (resp. $n y_{i}$ ). Thus the new net has still deficiency 0 .

Modelling the checking of the propositional formula. We now describe the subnet associated with the checking of propositional formula $\psi \equiv \bigwedge_{j \leq m} C_{j}$ where we assume w.l.o.g.: (1) that every clause $C_{j} \equiv l_{j, 1} \vee l_{j, 2} \vee l_{j, 3}$ has exactly three literals (i.e. variables or negated variables); and (2) that every variable or negated variable occurs at least in one clause. The left upper part of Figure 5 shows the Petri net which describes clause $C_{j}$ of the formula $\psi$. Places $\ell_{j, k}(k=1,2,3)$ represent the literals while places $n \ell_{j, k}$ represent the literal used as a proof of the clause, the place mutex $_{j}$ avoids to choose several proofs of the clause (and thus ensuring safeness), and finally place success $s_{j}$ can be marked if and only if the evaluation of the clause yields true for the current interpretation and one of its true literal is used as a proof.

The complexes of this subnet are mutex $_{j}+\ell_{j, k}$ (resp. success $s_{j}+n \ell_{j, k}$ ) with witness $-n \ell_{j, k}$ (resp. $n \ell_{j, k}$ ). So the subnet has deficiency 0 .

We now synchronise the clause subnets with the previous subnet in order to obtain the final net. Observe that in the previous subnet, transition $t_{0}$ (and $t_{0}^{\prime}$ ) must occur after every interpretation change. This is in fact the role of bit 0 of the counter. Thus we constrain its firing by requiring the places success $s_{j}$ to be marked as presented in the right upper part of Figure 5. Adding loops simply enlarges the complexes associated with $t_{0}$ and does not modify the incidence matrix. So the net has still deficiency 0.

It remains to synchronise the value of the variables and the values of the literals where the variables occur either positively or negatively. This is done in two steps. First $\ell_{j, k}$ is initially marked if the interpretation of the initial marking satisfies $\ell_{j, k}$. Then we synchronize the value changes as illustrated in the lower part of Figure 5. Once again the complexes are enlarged and the witnesses are still valid since the places $\ell_{j, k}$ do not belong to the support of any witness. 

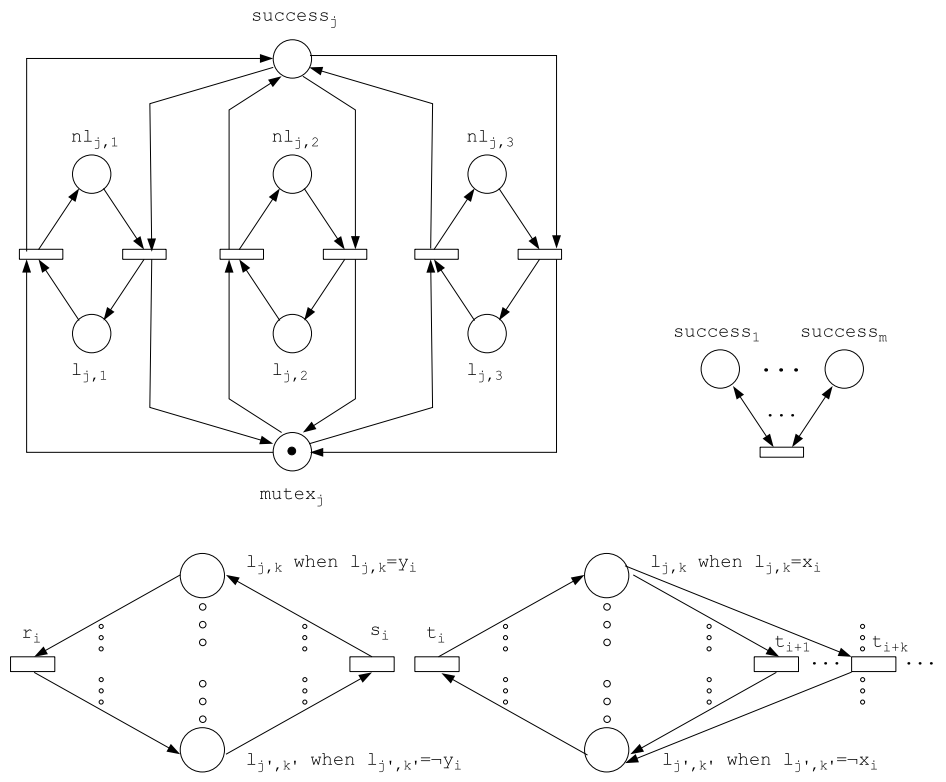

Fig. 5. Clause $C_{j}$ (left), synchronisation with $t_{0}$ (right) and with variables (below)

Choice of the initial and final marking for the net. Let us develop a bit the sequence $\bar{\sigma}_{n+3}$ in the two counter subnet in order to explain the choice of initial marking for this subnet: $\bar{\sigma}_{n+3}=\bar{\sigma}_{n+1} t_{n+1} t_{n+1}^{\prime} \bar{\sigma}_{n+1} t_{n+2} t_{n+2}^{\prime} \bar{\sigma}_{n+1} t_{n+1} t_{n+1}^{\prime} \bar{\sigma}_{n+1}$

We want to check all the interpretations of $x_{i}$ 's guessing the appropriate values of $y_{i}$ 's (if they exist). We have already seen that changing from one interpretation to another one (i.e. a counter incrementation or decrementation) allows to perform the allowed updates of $y_{i}$. However given the initial interpretation of the $x_{i}$ 's we need to make an initial guess of all the $y_{i}$ 's. So our initial marking restricted to the counter subnet will correspond to the marking reached after $\bar{\sigma}_{n+1} t_{n+1}$, i.e. corresponding to $c n t=2^{n+1}$ (i.e. word $010 \ldots 0$ ), $c n t^{\prime}=2^{n+1}-1$ (i.e. word $001 \ldots 1$ ) with in addition places $g o^{\prime}, u_{i}$ 's, mutex $_{j}$ 's and $y_{i}$ 's 1 -marked; places $\ell_{j, k}$ are marked according to the initial marking of places $x_{i}$ 's and $y_{i}$ 's as explained before. All the other places are unmarked. This explains the role of bit $n+1$.

Furthermore, if we have successfully checked all the interpretations of the $x_{i}$ 's, the counters will have reached the value $2^{n+2}-1$ (corresponding to a firing sequence obtained from $t_{n+1}^{\prime} \bar{\sigma}_{n+1}$ with possible updates of $y_{i}$ during change of interpretations). However we do not know what is the final guess for the $y_{i}$ 's. So firing transition $t_{n+2}$ allows to set the $y_{i}$ 's in such a way that the final marking will correspond to $c n t=2^{n+2}$ (i.e. word $10 \ldots 0$ ), $c n t^{\prime}=2^{n+2}-1$ (i.e. word $01 \ldots 1$ ) with in addition places $g o^{\prime}, u_{i}$ 's mutex $_{j}$ 's and $y_{i}$ 's 1-marked; places $\ell_{j, k}$ 
are marked accordingly. All the other places are unmarked. This explains the role of bit $n+2$.

By construction, the net reaches the final marking iff the formula is satisfied. Observe that the checking of clauses can be partially done concurrently with the change of interpretation. However as long as, in the net, a clause $C_{j}$ is "certified" by a literal $\ell_{j, k}$ (i.e. marking place success $s_{j}$ and unmarking place $\ell_{j, k}$ ) the value of the variable associated with the literal cannot change, ensuring that when $t_{0}$ is fired, the marking of any place success $_{j}$ corresponds to the evaluation of clause $C_{j}$ with the current interpretation.

Corollary 4.1. The liveness problem for safe $\Pi^{2}$-nets is PSPACE-complete.

Proof. Observe that the transitions of the net of the previous proof are fireable at least once (and so live by weak reversibility) iff $\varphi$ is true.

Let us now consider general (non-safe) Petri nets. Reachability and coverability of symmetric nets is EXPSPACE-complete [16]. In [9], it is proved that both problems are EXPSPACE-complete for WR nets (which include symmetric Petri nets). Next proposition establishes the same result for the coverability of $\Pi^{2}$-nets.

Proposition 4.1. The coverability problem for $\Pi^{2}$-nets is EXPSPACE-complete.

The complexity of reachability for $\Pi^{2}$-nets remains an open issue (indeed the proof of EXPSPACE-hardness does not work for reachability).

\section{The subclass of $\Pi^{3}$-nets}

In this section, we introduce $\Pi^{3}$-nets, a subclass of product-form Petri nets for which the normalising constant can be efficiently computed. The first subsection defines the subclass; the second one studies its structural properties and the third one is devoted to the computation of the normalising constant.

\subsection{Definition and properties}

Definition 5.1 (Ordered П-net) Consider an integer $n \geq 2$. An n-level ordered $\Pi$-net is a $\Pi$-net $\mathcal{N}=\left(P, T, W^{-}, W^{+}\right)$such that:

$$
\begin{aligned}
& \text { 1. } P=\underset{1 \leq i \leq n}{\bigsqcup} P_{i}, T=\bigsqcup_{1 \leq i \leq n} T_{i} \text { and } P_{i} \neq \emptyset \text { for all } 1 \leq i \leq n, \\
& \text { 2. } \mathcal{M}_{i}=\left(P_{i}, T_{i}, W_{\mid P_{i} \times T_{i}}^{-}, W_{\mid P_{i} \times T_{i}}^{+}\right) \text {is a strongly connected state machine, } \\
& \text { 3. } \forall 1 \leq i \leq n, \forall t \in T_{i}, \forall p \in P, \bullet t(p)>0 \text { implies } p \in P_{i} \text { or } p \in P_{i-1}\left(P_{0}=\emptyset\right) \text {, } \\
& \text { 4. } \forall 2 \leq i \leq n, \exists t \in T_{i}, \exists p \in P_{i-1} \text { s.t. } \bullet t(p)>0, \\
& \text { 5. } \forall 1 \leq i \leq n, \forall t, t^{\prime} \in T_{i},\left(\bullet \bullet \bullet^{\prime} t^{\prime}\right) \cap P_{i} \neq \emptyset \text { implies } \bullet=\bullet t^{\prime} . \\
& \text { We call } \mathcal{M}_{i} \text { the level } i \text { state machine. The elements of } P_{i} \text { (resp. } T_{i} \text { ) are level } \\
& i \text { places (resp. transitions). The complexes } \bullet \text { with } t \in T_{i} \text { are level } i \text { complexes. }
\end{aligned}
$$


By weak reversibility, the constraints 3, 4, and 5 also apply to the output bags $t^{\bullet}$. An ordered $\Pi$-net is a sequence of strongly connected state machines. Connections can only be made between a level $i$ transition and a level $(i-1)$ place (points 1, 2, 3). By construction, an ordered $\Pi$-net is connected (point 4). Each level $i$ place belongs to one and only one level $i$ complex (point 5).

Lemma 5.2 The reaction net of $\mathcal{N}$ is isomorphic to the disjoint union of state machines $\mathcal{M}_{i}$. So a $T$-semi-flow of $\mathcal{M}_{i}$ is also a $T$-semi-flow of $\mathcal{N}$. If a transition of $T_{i}$ is enabled by a reachable marking then every transition of $T_{i}$ is live.

An ordered П-net may be interpreted as a multi-level system. The transitions represent jobs or events while the tokens in the places represent resources or constraints. A level $i$ job requires resources from level $(i-1)$ and relocates these resources upon completion. Conversely, events occurring in level $(i-1)$ may make some resources unavailable, hence interrupting activities in level $i$. The dependency of an activity on the next level is measured by potentials, defined as follows.

Definition 5.3 (Interface, potential) A place $p \in P_{i}, 1 \leq i \leq n-1$, is an interface place if $p \in t^{\bullet}$ for some $t \in T_{i+1}$. For a place $p \in P_{i}, 2 \leq i \leq n$, and a place $q \in P_{i-1}$, set:

$$
\operatorname{pot}(p, q)= \begin{cases}t^{\bullet}(q) & \text { if } p \text { and } q \text { have a common input transition } t \in T_{i} \\ 0 & \text { otherwise }\end{cases}
$$

The potential of a place $p \in P_{i}, 2 \leq i$, is defined by: $\operatorname{pot}(p)=\sum_{q \in P_{i-1}} \operatorname{pot}(p, q)$. By convention, $\operatorname{pot}(p)=0$ for all $p \in P_{1}$.

By the definition of ordered $\Pi$-nets, the quantity $t^{\bullet}(q)$ does not depend on the choice of $t$, so the potential is well-defined.

Example. The Petri net in Figure 6 is a 3-level ordered $\Pi$-net. The potentials are written in parentheses. To keep the figure readable, the arcs between the place $p_{1}$ and the level 2 transitions are omitted.

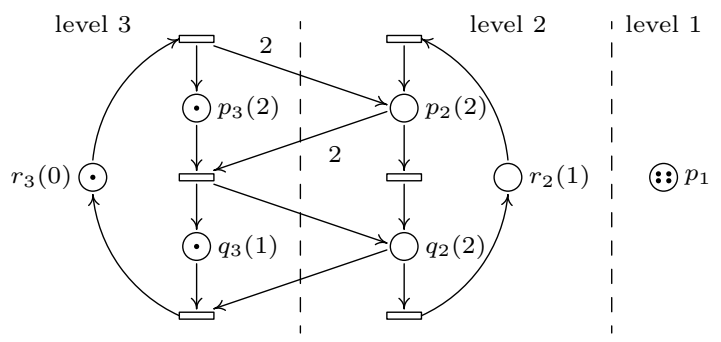

Fig. 6. Ordered П-net. 
The behaviour of the state machines $\mathcal{M}_{i}$ is embedded in the behaviour of $\mathcal{N}$, in the sense that the marking stripped off of the potentials evolves like a marking of the state machines.

Definition 5.4 (Effective marking) The effective marking of a marking $m$, denoted by $\widetilde{m}$, is defined as follows. For all $i \leq n$ and $p \in P_{i}$,

$$
\widetilde{m}(p)=m(p)+\sum_{j=1}^{n-i}\left((-1)^{j} \sum_{\substack{r_{1} \in P_{i+1} \\ r_{j} \in \dddot{P}_{i+j}}} m\left(r_{j}\right)\left(\prod_{k=1}^{j-1} \operatorname{pot}\left(r_{k+1}, r_{k}\right)\right) \operatorname{pot}\left(r_{1}, p\right)\right) .
$$

Remark. Note that an effective marking is not necessarily non-negative. It can be showed by induction that:

$\forall p \in P_{n}, \widetilde{m}(p)=m(p)$ and $\forall p \in P_{i}, i<n, \widetilde{m}(p)=m(p)-\sum_{r \in P_{i+1}} \tilde{m}(r) \operatorname{pot}(r, p)$

Lemma 5.5 Let $m, m^{\prime}$ be two vectors such that $m^{\prime}=m+W(t)$ for some $t \in T_{i}(1 \leq i \leq n)$. Let $p_{1}$ and $p_{2}$ denote the input place and the output place of $t$ in $P_{i}$, respectively. Then for every place $p$ :

$$
\widetilde{m}^{\prime}(p)=\widetilde{m}(p)-1 \text { if } p \text { is } p_{1}, \quad \widetilde{m}(p)+1 \text { if } p \text { is } p_{2}, \quad \widetilde{m}(p) \text { otherwise. }
$$

The above lemma applies in particular when $m$ and $m^{\prime}$ are markings such that $m \stackrel{t}{\longrightarrow} m^{\prime}$. Eqns (5.2) look like the equations for witnesses. Since each level $i$ complex contains exactly one level $i$ place, one guesses that every complex admits a witness, i.e. that $\mathcal{N}$ is a $\Pi^{2}$-net. This is confirmed by the next proposition.

Proposition 5.6 Let $B$ denote the $P \times P$ integer matrix of the linear transformation $m \mapsto \widetilde{m}$ defined by (5.1). For $p \in P_{i}$, the line vector $B(p)$ is a witness for the $i$-level complex containing $p$. In particular, $\mathcal{N}$ is a $\Pi^{2}$-net.

Lemma 5.5 allows to derive the S-invariants of $\mathcal{N}$ induced by $\mathrm{S}$-semi-flows.

Corollary 5.7 Let $m_{0}$ be the initial marking of $\mathcal{N}$. We have:

$$
\forall m \in \mathcal{R}\left(m_{0}\right), \quad \forall i \in\{1, \ldots, n\}, \quad \widetilde{m}\left(P_{i}\right)=\widetilde{m}_{0}\left(P_{i}\right)
$$

More generally, for all $i$, the vector $v_{i}=\sum_{p \in P_{i}} B(p)$ is a S-semi-flow of $\mathcal{N}$.

Example. Consider the ordered $\Pi$-net in Figure 6 with the initial marking $m_{0}=$ $p_{3}+q_{3}+r_{3}+4 p_{1}$. The effective marking of $m_{0}$ is $\widetilde{m_{0}}=p_{3}+q_{3}+r_{3}-2 p_{2}-q_{2}+10 p_{1}$. Any reachable marking $m$ satisfies the invariants:

$$
\begin{gathered}
m\left(P_{3}\right)=3 \\
m\left(P_{2}\right)-2 m\left(p_{3}\right)-m\left(q_{3}\right)=-3 \\
m\left(p_{1}\right)-2 m\left(p_{2}\right)-2 m\left(q_{2}\right)-m\left(r_{2}\right)+4 m\left(p_{3}\right)+2 m\left(q_{3}\right)=10
\end{gathered}
$$

It can be shown that $\left\{v_{i}, 1 \leq i \leq n\right\}$ is a basis of the S-semi-flows of $\mathcal{N}$.

Proposition 5.8 Let $v$ be an $S$-semi-flow of $\mathcal{N}$, i.e. $v . W=0$. There exist rational numbers $a_{1}, \ldots, a_{n}$ such that $v=\sum_{i=1}^{n} a_{i} v_{i}$. 
The independence of the set $\left\{v_{i}, 1 \leq i \leq n\right\}$ follows from the fact that the vectors $v_{i} B^{-1}$ have non-empty disjoint supports.

We now consider only ordered $\Pi$-nets in which the interface places in $P_{i}$ have maximal potential among the places of $P_{i}$. From the technical point of view, this assumption is crucial for the reachability set ananlysis presented later. From the modelling point of view, it is a reasonable restriction. Consider the multi-level model, the assumption means that during the executions of level $i$ jobs, the level $(i-1)$ is idle, therefore the amount of available resource is maximal.

Definition $5.9\left(\Pi^{3}\right.$-net) An ordered $\Pi$-net $\mathcal{N}$ is a $\Pi^{3}$-net if:

$$
\forall i, \forall p \in P_{i}: p \in{ }^{\bullet} T_{i+1} \Longrightarrow \operatorname{pot}(p)=\max \left\{\operatorname{pot}(q), q \in P_{i}\right\} \text {. }
$$

\subsection{The reachability set}

From now on, $\mathcal{N}$ is a $n$-level $\Pi^{3}$-net with $\mathcal{M}_{1}, \ldots, \mathcal{M}_{n}$ its state machines.

Definition 5.10 (Minimal marked potential) Consider $i \in\{2, \ldots, n\}$. The level $i$ minimal potential marked by $m$ is:

$$
\varphi_{i}(m)= \begin{cases}\max \left\{\operatorname{pot}(p), p \in P_{i}\right\} & \text { if } m\left(P_{i}\right)=0, \\ \min \left\{\operatorname{pot}(p), p \in P_{i}, m(p)>0\right\} & \text { if } m\left(P_{i}\right)>0 .\end{cases}
$$

Next lemma gives a necessary condition for reachability.

Lemma 5.11 If $\varphi_{i}(m) \leq m\left(P_{i-1}\right)$ then $\varphi_{i}\left(m^{\prime}\right) \leq m^{\prime}\left(P_{i-1}\right)$ for all $m^{\prime} \in \mathcal{R}(m)$.

For our purposes, we now define the partial liveness and partial reachability.

Definition 5.12 ( $i$-reachability set, $i$-liveness) Let $m$ be a marking. The $i$ reachability set of $m$, denoted by $\mathcal{R}_{i}(m)$, is the set of all markings reachable from $m$ by a firing sequence consisting of transitions in $\bigcup_{1 \leq j \leq i} T_{j}$. We say that $m$ is $i$-live if for any transitions $t$ in $\bigcup_{1 \leq j \leq i} T_{j}$, there exists a marking in $\mathcal{R}_{i}(m)$ which enables t. By convention, $\mathcal{R}_{0}(m)=\{m\}$ and every marking is 0-live.

The $i$-live markings are characterised by the following proposition.

Proposition 5.13 A marking $m$ is $i$-live if and only if it satisfies the following inequalities, called the $i$-condition:

$$
m\left(P_{i}\right)>0 \wedge \forall 2 \leq j \leq i: m\left(P_{j-1}\right) \geq \varphi_{j}(m)
$$

If $m$ satisfies the $i$-condition then for every $p, q \in P_{i}$ such that $p \neq q, m(p)>0$ and $\operatorname{pot}(p) \leq m\left(P_{i-1}\right)$, there exists $m^{\prime} \in \mathcal{R}_{i}(m)$ such that:

$$
m^{\prime}(p)=m(p)-1, m^{\prime}(q)=m(q)+1, \forall r \in P_{i} \backslash\{p, q\}, m^{\prime}(r)=m(r) .
$$

A marking is live if and only if it satisfies the $n$-condition. 
Example: The ordered $\Pi$-net in Figure 6 is a $\Pi^{3}$-net. Consider two markings: $m_{1}=p_{3}+q_{3}+r_{3}+4 p_{1}$ and $m_{2}=3 q_{3}+4 p_{1}$. These markings agree on all the S-invariants, but only $m_{1}$ satisfies the 3 -condition. It is easy to check that $m_{1}$ is live while $m_{2}$ is dead.

We conclude this subsection by showing that the reachability problem for $\Pi^{3}$-nets can be efficiently decided as well.

Theorem 5.14 Suppose that the initial marking $m_{0}$ is live. Then the reachability set $\mathcal{R}\left(m_{0}\right)$ coincides with the set $\mathcal{S}\left(m_{0}\right)$ of markings which satisfy the $n$-condition and agree with $m_{0}$ on the S-invariants given by Corollary 5.7.

\subsection{Computing the normalising constant}

The normalising constant of a product-form Petri net (see Section 2.1) is $G=$ $\sum_{m} \mathbb{1}_{m \in \mathcal{R}\left(m_{0}\right)} \prod_{p \in P} u_{p}^{m(p)}$. It is in general a difficult task to compute $G$, as can be guessed from the complexity of the reachability problem. However, efficient algorithms may exist for nets with a well-structured reachability set. Such algorithms were known for Jackson networks [18] and the $S$-invariant reachable Petri nets defined in [6]. We show that is is also the case for the class of live $\Pi^{3}$-nets which is strictly larger than the class of Jackson networks (which correspond to 1-level ordered nets) and is not included in the class of S-invariant reachable Petri nets.

Suppose that $m_{0}$ is a live marking. Suppose that the places of each level are ordered by increasing potential: $P_{i}=\left\{p_{i 1}, \ldots, p_{i k_{i}}\right\}$ such that $\forall 1 \leq j<k_{i}$, $\operatorname{pot}\left(p_{i j}\right) \leq \operatorname{pot}\left(p_{i(j+1)}\right)$.

Let $V$ denote the $n \times P$-matrix the $i$-th row of which is the S-invariant $v_{i}$ defined in Corollary 5.7. For $1 \leq i \leq n$, set $C_{i}=v_{i} m_{0}=\widetilde{m}_{0}\left(P_{i}\right)$. Then the reachability set consists of all $n$-live markings $m$ such that $V m={ }^{t}\left(C_{1}, \ldots, C_{n}\right)$.

For $1 \leq i \leq n, 1 \leq j \leq k_{i}$ and $c_{1}, \ldots, c_{i} \in \mathbb{Z}$, define $E\left(i, j, c_{1}, \ldots, c_{i}\right)$ as the set of markings $m$ such that

$$
\left\{\begin{array}{l}
m\left(p_{i \nu}\right)=0 \text { for all } \nu>j \\
V m={ }^{t}\left(c_{1}, \ldots, c_{i}, 0 \ldots, 0\right) \\
\varphi_{\nu}(m) \leq m\left(P_{\nu-1}\right) \text { for all } 2 \leq \nu \leq i
\end{array}\right.
$$

The elements of $E\left(i, j, c_{1}, \ldots, c_{i}\right)$ are the markings which satisfy the second part of the $i$-condition and the S-invariants constraints $\left(c_{1}, \ldots, c_{i}, 0, \ldots, 0\right)$ and concentrate tokens in $P_{1}, \ldots, P_{i-1}$ and $\left\{p_{i 1}, \ldots, p_{i j}\right\}$.

With each $E\left(i, j, c_{1}, \ldots, c_{i}\right)$ associate

$$
G\left(i, j, c_{1}, \ldots, c_{i}\right)=\pi\left(E\left(i, j, c_{1}, \ldots, c_{i}\right)\right)=\sum \prod_{p \in P} u_{p}^{m(p)}
$$

the sum being taken over all $m \in E\left(i, j, c_{1}, \ldots, c_{i}\right)$.

We propose to compute $G\left(n, k_{n}, C_{1}, \ldots, C_{n}\right)$ by dynamic programming. It consists in breaking each $G\left(i, j, c_{1}, \ldots, c_{i}\right)$ into smaller sums. This corresponds to a partition of the elements of $E\left(i, j, c_{1}, \ldots, c_{i}\right)$ by the number of tokens in $p_{i j}$. 
Proposition 5.15 Let be given $E=E\left(i, j, c_{1}, \ldots, c_{i}\right)$. If $c_{i}<0$ then $E=\emptyset$. If $c_{i} \geq 0$ then for every non-negative integer $a$ :

1. If $a>c_{i}$ then $E \cap\left\{m \mid m\left(p_{i j}\right)=a\right\}=\emptyset$.

2. If $a<c_{i}$ and $j=1$ then $E \cap\left\{m \mid m\left(p_{i j}\right)=a\right\}=\emptyset$.

3. If $a<c_{i}$ and $j \geq 2$ then $E \cap\left\{m \mid m\left(p_{i j}\right)=a\right\}=E\left(i, j-1, c_{1}-v_{1}\left(a p_{i j}\right), \ldots, c_{i}-\right.$ $\left.v_{i}\left(a p_{i j}\right)\right)$ :

4. If $a=c_{i}$ and $i=1$ then $E \cap\left\{m \mid m\left(p_{i j}\right)=a\right\}=\left\{c_{1} p_{1 j}\right\}$.

5. If $a=c_{i}$ and $i>1$ then $E \cap\left\{m \mid m\left(p_{i j}\right)=a\right\}=E\left(i-1, k_{i-1}, c_{1}-\right.$ $\left.v_{1}\left(a p_{i j}\right), \ldots, c_{i-1}-v_{i-1}\left(a p_{i j}\right)\right)$ :

Proposition 5.15 induces the following relations between the sums $G\left(i, j, c_{1}, \ldots, c_{i}\right)$.

Corollary 5.16 If $c_{i}<0$ then $G\left(i, j, c_{1}, \ldots, c_{i}\right)=0$. If $c_{i} \geq 0$ then:

- Case $2 \leq i \leq n, 2 \leq j \leq k_{i}$ :

$$
\begin{aligned}
G\left(i, j, c_{1}, \ldots, c_{i}\right)= & \sum_{\nu=0}^{c_{i}-1} u_{p_{i j}}^{\nu} G\left(i, j-1, c_{1}-v_{1}\left(\nu p_{i j}\right), \ldots, c_{i}-v_{i}\left(\nu p_{i j}\right)\right) \\
& +u_{p_{i j}}^{c_{i}} G\left(i-1, k_{i-1}, c_{1}-v_{1}\left(c_{i} p_{i j}\right), \ldots, c_{i-1}-v_{i-1}\left(c_{i} p_{i j}\right)\right) .
\end{aligned}
$$

- Case $2 \leq i \leq n, j=1$ :

$$
\begin{aligned}
& G\left(i, 1, c_{1}, \ldots, c_{i}\right)=u_{p_{i 1}}^{c_{i}} G\left(i-1, k_{i-1}, c_{1}-v_{1}\left(c_{i} p_{i 1}\right), \ldots, c_{i-1}-v_{i-1}\left(c_{i} p_{i 1}\right)\right) . \\
& - \text { Case } i=1, j \geq 2: G\left(1, j, c_{1}\right)=\sum_{\nu=0}^{c_{1}-1} u_{p_{1 j}}^{\nu} G\left(1, j-1, c_{1}-\nu\right)+u_{p_{1 j}}^{c_{1}} . \\
& - \text { Case } i=1, j=1: G\left(1,1, c_{1}\right)=u_{p_{11}}^{c_{1}} .
\end{aligned}
$$

Complexity. Since $i \leq n, j \leq K=\max \left\{k_{1}, \ldots, k_{n}\right\}$, the number of evaluations is bounded by $n \times K \times \gamma$, where $\gamma$ upper bounds the $c_{i}$ 's. Let $\alpha$ denote the global maximal potential. From (5.1), we obtain $\gamma=\mathcal{O}\left(m_{0}(P) K^{n} \alpha^{n}\right)$. So the complexity of a dynamic programming algorithm using Cor. 5.16 is $\mathcal{O}\left(m_{0}(P) n K^{n+1} \alpha^{n}\right)$, i.e. pseudo-polynomial for a fixed number of state machines.

\section{Perspectives}

This work has several perspectives. First, we are interested in extending and applying our rules for a modular modelling of complex product-form Petri nets. Then we want to validate the formalism of $\Pi^{3}$-nets showing that it allows to express standard patterns of distributed systems. Finally we conjecture that reachability is EXPSPACE-complete for $\Pi^{2}$-nets and we want to establish it.

Acknowledgements. We would like to thank the anonymous referess whose numerous and pertinent suggestions have been helpful in preparing the final version of the paper. 


\section{References}

[1] M. Ajmone Marsan, G. Balbo, G. Conte, S. Donatelli, G. Franceschinis. Modelling with Generalized Stochastic Petri Nets. John Wiley \& Sons, 1995.

[2] F. Baccelli, G. Cohen, G.J. Olsder, and J.P. Quadrat. Synchronization and Linearity. John Wiley \& Sons, New York, 1992.

[3] F. Baskett, K. M. Chandy, R. R. Muntz, F. Palacios. Open, closed and mixed networks of queues with different classes of customers. Journal of the ACM, 22(2):248-260, April 1975.

[4] R. J. Boucherie, M. Sereno. On closed support T-invariants and traffic equations. Journal of Applied Probability, (35): 473-481, 1998.

[5] J. Esparza and M. Nielsen. Decidability issues for Petri nets - a survey. Journal of Informatik Processing and Cybernetics, 30(3):143-160, 1994.

[6] J.L. Coleman, W. Henderson, P.G. Taylor. Product form equilibrium distributions and a convolution algorithm for stochastic Petri nets. Performance Evaluation, 26(3):159-180, September 1996.

[7] J. Esparza. Reduction and Synthesis of Live and Bounded Free Choice Petri Nets. Information and Computation, 114(1):50-87, 1994

[8] M. Feinberg. Lectures on chemical reaction networks. Given at the Math. Research Center, Univ. Wisconsin, 1979. Available online at http://www. che.eng. ohio-state.edu/ feinberg/LecturesOnReactionNetworks.

[9] S. Haddad, P. Moreaux, M. Sereno, M. Silva Product-form and stochastic Petri nets: a structural approach. Performance Evaluation, 59: 313-336, 2005.

[10] W. Henderson, D. Lucic, P.G. Taylor. A net level performance analysis of stochastic Petri nets. Journal of Australian Mathematical Soc. Ser. B, 31:176-187, 1989.

[11] J. R. Jackson. Jobshop-like Queueing Systems. Management Science, 10(1): 131142, 1963.

[12] F. Kelly. Reversibility and Stochastic Networks. Wiley, New-York, 1979.

[13] A. A. Lazar, T. G.Robertazzi. Markovian Petri Net Protocols with Product Form Solution. Proc. of INFOCOM 87, pp. 1054-1062, San Francisco, CA, USA, 1987.

[14] M. Li, N. D. Georganas. Parametric Analysis of Stochastic Petri Nets, Fifth International Conference on Modelling and Tools for Computer Performance Evaluation, Torino, Italy, 1991,

[15] J. Mairesse, H-T. Nguyen. Deficiency Zero Petri Nets and Product Form. Petri Nets 2009, LNCS 5606, 103-122, 2009.

[16] E. Mayr, A. Meyer. The complexity of the word problem for commutative semigroups an polynomial ideals. Advances in Math, 46 (1982), 305-329.

[17] C. Papadimitriou. Computational Complexity. Addison Wesley, 1994.

[18] M. Reiser, S.S. Lavenberg. Mean Value Analysis of Closed Multichain Queueing Networks. Journal of the ACM, 27(2): 313-322, 1980. 